\section{Intermittent abdominal pain due to pancreatic pseudocyst hemorrhage diagnosed by endoscopic ultrasound}

A 36-year-old man presented to the emergency room with a 3-week history of intermittent, intense epigastric pain. He had an episode of alcoholic pancreatitis 8 months before, which was complicated by a 9-cm pseudocyst located in the body and tail of the pancreas. The patient did not attend the follow-up appointment. He denied having fever, vomiting, weight loss, or gastrointestinal hemorrhage, and claimed to be abstemious. Physical examination was normal, except for epigastric tenderness. Blood tests including hemogram, amylase, lipase, and inflammatory markers were unremarkable. Computed tomography (CT) scan showed that the pseudocyst remained stable in size and contained a small amount of high-at- tenuation debris ( $\bullet$ Fig. 1 ); there were signs of left portal hypertension determined by compression of the splenic vein. Endoscopic ultrasound (EUS) revealed a vessel within the cyst, evidence of intracystic hemorrhage, and hemosuccus pancreaticus ( $\bullet$ Fig.2). The patient remained hemodynamically stable but his hemoglobin level dropped by $1.5 \mathrm{~g} / \mathrm{dL}$. No pseudoaneurysm or contrast extravasation was detected on abdominal angiography. Emergent surgery was proposed, but the patient refused further intervention and was discharged on request. A few weeks later, the patient returned to the emergency room due to abdominal pain. CT demonstrated a vessel within the cystic wall ( Fig.3). The patient under-

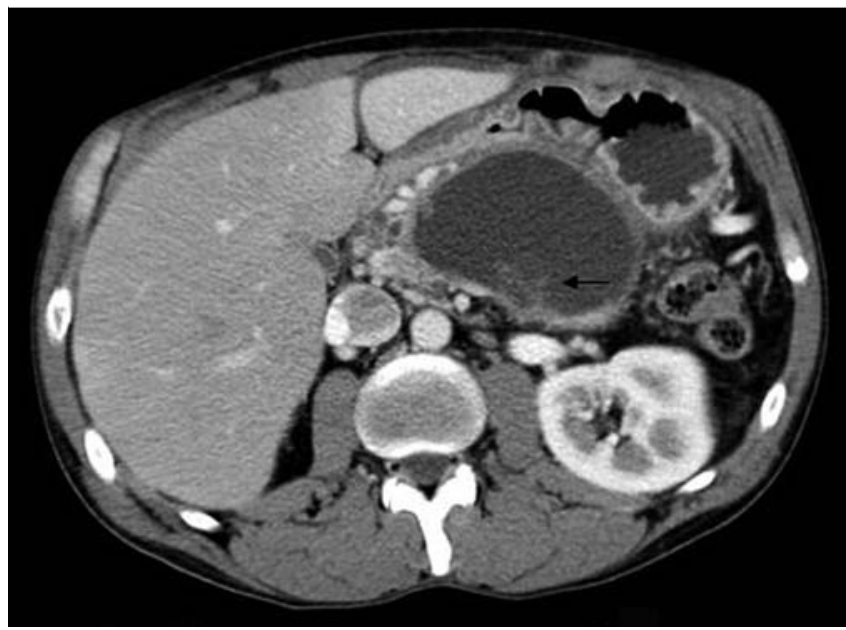

Fig. 1 Initial contrastenhanced abdominal computed tomography (CT) scan in a 36-yearold man with a 3-week history of intermittent, intense epigastric pain: the pseudocyst is seen as a thick-walled, unilocular, low-attenuation fluid collection with a high-attenuation area within the cavity (arrow).
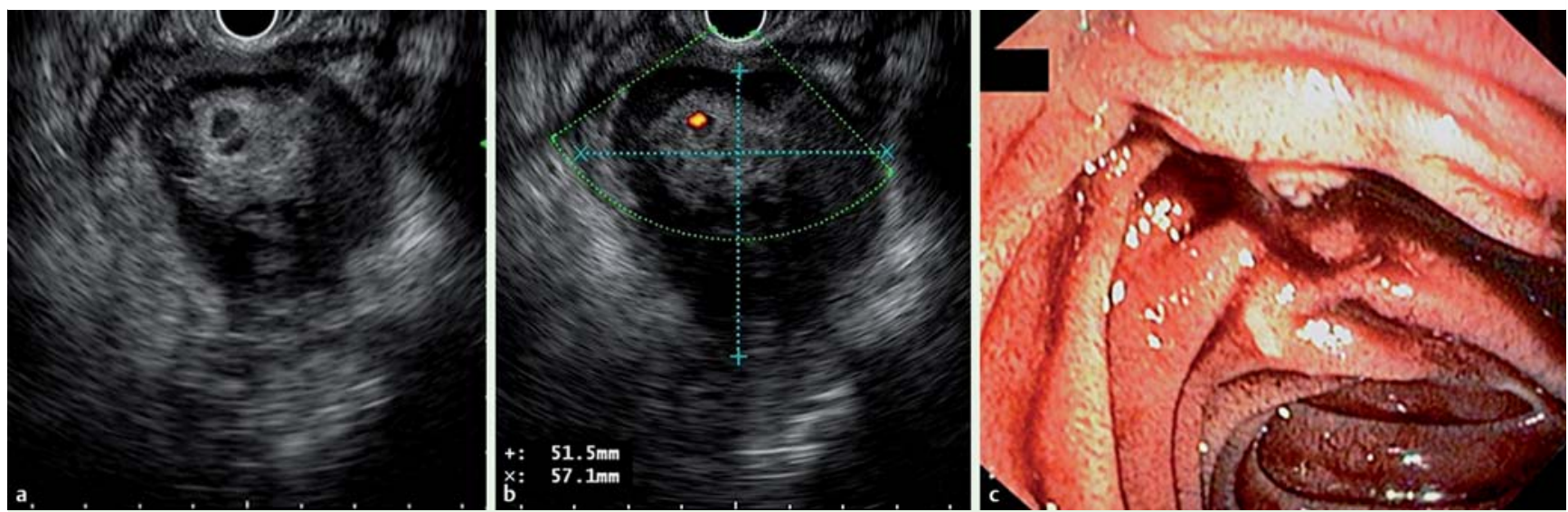

Fig. 2 a Endoscopic ultrasound (EUS) view showing a vascular structure inside the pseudocyst and the inhomogeneous, echo-dense cystic contents due to intracystic hemorrhage. $\mathbf{b}$ The blood flow signal was confirmed by power Doppler. $\mathbf{c}$ During EUS examination, fresh blood was observed in the duodenal lumen, with active bleeding from the papilla of Vater (hemosuccus pancreaticus). 


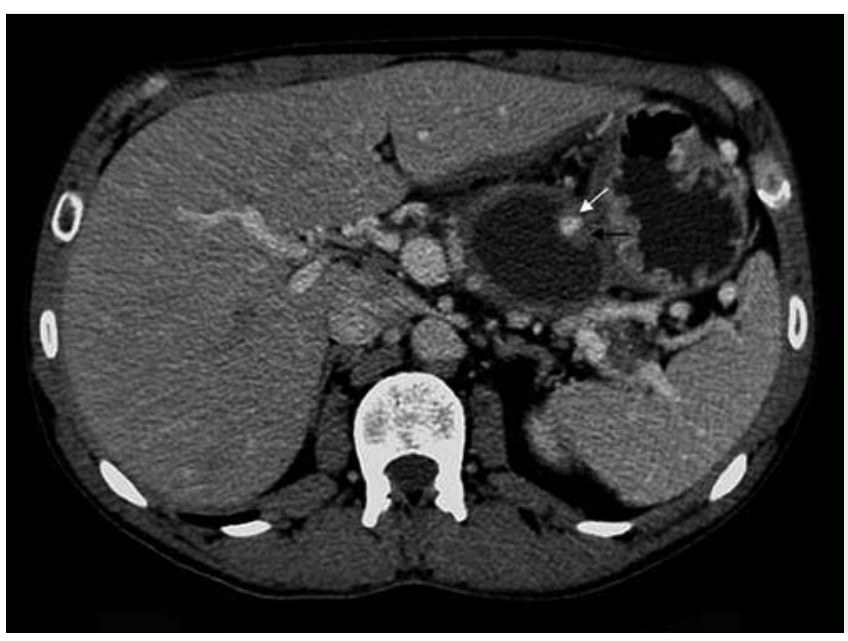

Fig. 3 Abdominal computed tomography (CT) scan demonstrating a vessel within the thickened wall of the pseudocyst. There is a fresh hyperdense thrombus inside the vessel (black arrow) with the patent lumen filled with contrast material (white arrow). There was no evidence of active bleeding.

\section{Bibliography}

Dol http://dx.doi.org/

10.1055/s-0033-1344769

Endoscopy 2013; 45: E367-E368

(c) Georg Thieme Verlag KG

Stuttgart · New York

ISSN 0013-726X

\section{Corresponding author}

\section{G. Rodrigues}

Fernando Fonseca Hospital - Gastroenterology IC19 Venteira Amadora

Lisbon 2720-276

Portugal

Fax: +351-214-345566

catarinagr@hotmail.com 